\title{
Towards the Total Synthesis of Aerucyclamide B
}

\section{Stella Peña, Laura Scarone, Gloria Serra*}

Departamento de Química Orgánica, Facultad de Química, UdelaR, Gral. Flores 2124, Montevideo, Uruguay

\author{
*gserra@fq.edu.uy
}

Keywords: macrocycles, antimalarial, heterocycles

\section{INTRODUCTION}

Aerucyclamides $A, B, C$ and $D$ were isolated from the cyanobacteria Microcystis aeruginosa PCC 7806. Aerucyclamide B (1), Figure 1, shows the highest biological activity of the four against Plasmodium falciparum.

This compound class encompasses hexacyclopeptides alternating in hydrophobic and hydrophilic (Ser, Thr, and Cys) amino acids. The side chains of these polar amino acids are heterocyclized to form oxazole or thiazole rings or their reduced derivatives.

As part of our search for antiparasitic agents, ${ }^{2}$ in the present work we report the results towards the total synthesis of Aerucyclamide B.

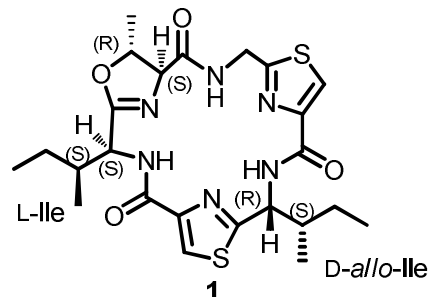

Figure 1. Aerucyclamide $B$

\section{RESULTS AND DISCUSSION}

In order to obtain Aerucyclamide B, building blocks 2, 3, and 4, Scheme 1, were prepared. The dipeptide 2 was synthesized from Boc-L-Ile-OH and L-allo-Thr-OMe using HBTU as coupling reagent.

Thiazole $\mathbf{3}$ was obtained by Hantzsch reaction from Boc-Gly thioamide and ethyl bromopyruvate in good yield $(65 \%)$.

Starting from Boc-D-allo-lle-OH and L-Ser-OMe, the corresponding oxazoline was obtained using coupling reaction and then cyclodehydration. ${ }^{3}$ Thiolysis of the oxazoline provided the corresponding thiomide. ${ }^{4}$ Finally, thiazole 4 was prepared from the obtained thioamide by cyclodehydration and oxidation processes using DAST and $\mathrm{BrCCl}_{3} / \mathrm{DBU}$ in $90 \%$ yield.

Coupling reaction between the deprotected $\mathbf{3}$ and $\mathbf{4}$ rendered the corresponding bis heterocycle. The transformation to the macrocycle $\mathbf{5}$ and the cyclodehydration reaction to obtain Aerucyclamide B (1) are under investigation in our laboratory.
Scheme 1. Synthesis of Aerucyclamide B

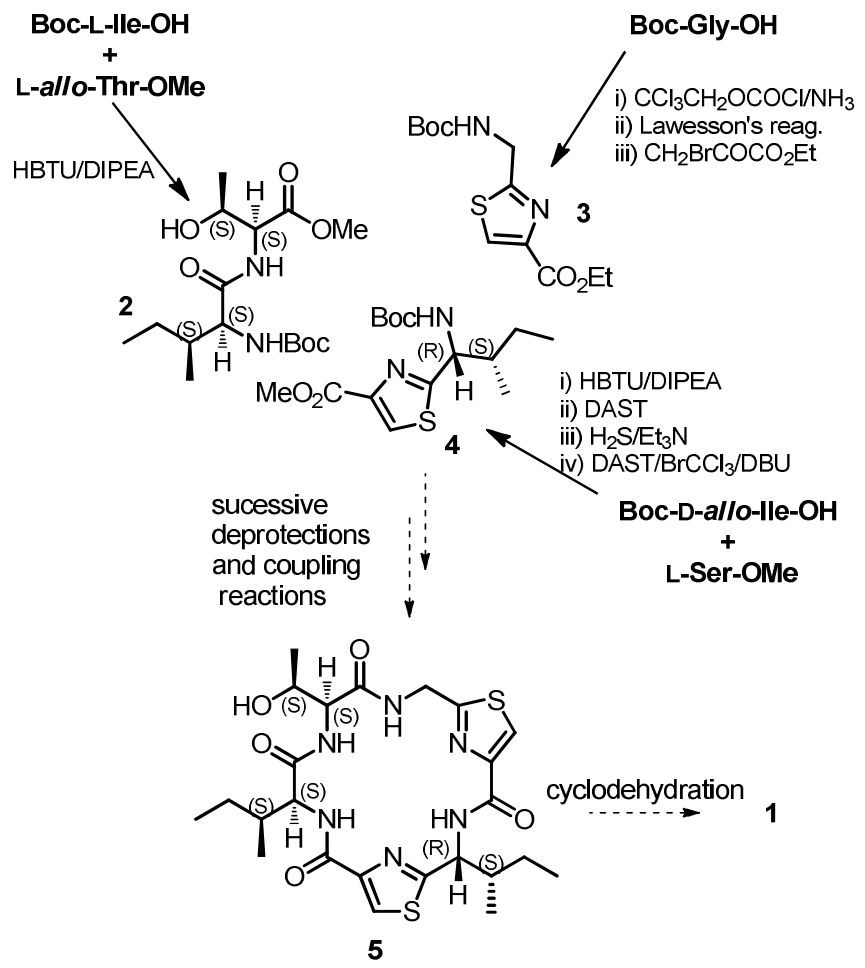

\section{CONCLUSION}

Key fragments to the synthesis of Aerucyclamide B were obtained in good yield. Biological evaluation of some of the obtained products and optimization of the reaction conditions to the synthesis of macrocycles $\mathbf{5}$ and $\mathbf{1}$ is currently in progress.

\section{ACKNOWLEDGEMENTS}

ANII(FCE_2720),CSIC Grupos, PEDECIBA, UdelaR

\section{REFERENCES}

${ }^{1}$ a) Portmann, C.; Blom, J. F.; Gademann, K.; Jüttner, F. J. Nat. Prod. 2008, 71, 1193. b) Portmann, C., Blom, J. F., Kaiser, M., Brun, R., Jüttner, F. Gademann, K. J. Nat. Prod. 2008, 71, 1891.

${ }^{2}$ a) Sellanes, D.; Campot, F.; Nuñez, I.; Lin, G.; Espósito, P.; Saldaña, J.; Domínguez, L.; Manta, E.; Serra, G. Tetrahedron 2010, 66, 5384. b) Scarone, L.; Fajardo, J.; Saldaña, J.; Domínguez, L.; Espósito, P.; Dematteis, S.; Wipf, P.; Manta, E.; Serra, G. Lett. Drug, Des. \& Disc. 2009, 6, 413.

${ }^{3}$ Phillips, A.J.; Yoshikazi, U.; Wipf, P.; Reno, M.J.; Williams, D.R. Org Lett. 2000, 2, 1165.

4 Wipf, P.; Miller, C. P.; Venkatraman, S.; Fritch, P. C.

Tetrahedron Lett. 1995, 36, 6395. 